\title{
Electroweak probes in heavy-ion collisions with ATLAS
}

\author{
Jakub Kremer*on behalf of the ATLAS Collaboration \\ Johannes Gutenberg-Universität Mainz, \\ Institut für Physik, \\ Staudingerweg 7, 55128 Mainz, Germany \\ E-mail: jakub.kremer@cern.ch
}

\begin{abstract}
Electroweak bosons produced in lead-lead $(\mathrm{Pb}+\mathrm{Pb})$ collisions are an excellent tool to constrain initial-state effects which affect the rates of hard-scattering processes in nucleus-nucleus interactions. The production yields of massive electroweak bosons, observed via their leptonic decay channels, offer a high-precision test of the binary collision scaling expected in $\mathrm{Pb}+\mathrm{Pb}$ and a way to quantify nuclear modifications of the parton distribution functions (PDFs). The large samples of $\mathrm{Pb}+\mathrm{Pb}$ data at $\sqrt{s_{\mathrm{NN}}}=5.02 \mathrm{TeV}$ collected by the ATLAS experiment in 2015, and the corresponding high-statistics $p p$ data at the same collision energy used as a baseline, allow for a detailed experimental study of these phenomena and comparisons to predictions from a variety of theoretical calculations. This report presents the latest ATLAS results on electroweak boson production, including updated results on $Z$ production and high-precision $W$ boson results in $\mathrm{Pb}+\mathrm{Pb}$ collisions. Inclusive production of prompt photons in proton-lead $(p+\mathrm{Pb})$ collisions at $\sqrt{s_{\mathrm{NN}}}=8.16 \mathrm{TeV}$ is also covered. Various predictions of nuclear modifications to PDFs are discussed.
\end{abstract}

40th International Conference on High Energy physics - ICHEP2020

July 28 - August 6, 2020

Prague, Czech Republic (virtual meeting)

\footnotetext{
${ }^{*}$ Speaker
} 


\section{Introduction}

Measurements of electroweak boson production in proton-proton $(p p)$ collisions are powerful tests of the Standard Model of particle physics, since they rely on precise calculations based on QCD, including a good knowledge of parton distribution functions (PDFs), and the electroweak theory. In the context of ultrarelativistic heavy-ion collisions, they provide typically a precise reference for measurements of electroweak boson production in proton-nucleus or nucleus-nucleus collisions. By comparing results from proton-nucleus collisions to those from $p p$ collisions, one can study cold nuclear matter effects including nuclear modifications of PDFs (nPDFs) or the energy loss of partons in the initial state. Nucleus-nucleus collisions at LHC energies produce a strongly interacting quark-gluon plasma (QGP), which leads to final-state effects such as jet quenching. However, electroweak bosons or their leptonic decay products do not carry a colour charge, so that they are not affected significantly by interactions with the QGP. Therefore, measurements of electroweak boson production provide an excellent tool to verify models describing the geometry and centrality of heavy-ion collisions.

In order to quantify modifications of electroweak boson production in proton-nucleus collisions, the nuclear modification factor, $R_{p \mathrm{~A}}$, is defined as:

$$
R_{p \mathrm{~A}}=\frac{1}{A} \frac{\sigma_{p+\mathrm{A}}}{\sigma_{p p}}
$$

where $A$ is the mass number of the nucleus, while $\sigma_{p+\mathrm{A}}$ and $\sigma_{p p}$ are cross-sections for electroweak boson production in proton-nucleus and $p p$ collisions, respectively, measured at the same centreof-mass energy.

For nucleus-nucleus collisions, the collision geometry has to be taken into account when studying modifications of electroweak boson production. The corresponding nuclear modification factor, $R_{\mathrm{AA}}$, is defined as:

$$
R_{\mathrm{AA}}=\frac{N_{\mathrm{AA}} / N_{\mathrm{evt}}}{\left\langle T_{\mathrm{AA}}\right\rangle \cdot \sigma_{p p}}
$$

Here, $N_{\mathrm{AA}} / N_{\mathrm{evt}}$ is the electroweak boson yield measured per minimum-bias nucleus-nucleus collision, and $\sigma_{p p}$ is the corresponding cross-section measured in $p p$ collisions at the same centre-ofmass energy. The average nuclear thickness function, $\left\langle T_{\mathrm{AA}}\right\rangle$, encodes the geometry of the collision, and typically the observable $\frac{N_{\mathrm{AA}} / N_{\mathrm{evt}}}{\left\langle T_{\mathrm{AA}}\right\rangle}$ is referred to as the normalised production yield.

This report presents measurements of electroweak boson production using data collected by the ATLAS experiment [1] at the Large Hadron Collider (LHC). In particular, it is focused on a measurement of prompt photon production in proton-lead $(p+\mathrm{Pb})$ collisions at $\sqrt{s_{\mathrm{NN}}}=8.16 \mathrm{TeV}$ [2] and measurements of $W$ and $Z$ boson production in $p p$ collisions at $\sqrt{s}=5.02 \mathrm{TeV}$ [3] and leadlead $(\mathrm{Pb}+\mathrm{Pb})$ collisions at $\sqrt{s_{\mathrm{NN}}}=5.02 \mathrm{TeV}[4,5]$.

\section{Measurement of prompt photon production in $p+\mathrm{Pb}$ collisions}

The measurement of prompt photon production in $p+\mathrm{Pb}$ collisions at $\sqrt{s_{\mathrm{NN}}}=8.16 \mathrm{TeV}$ is based on events selected using single-photon triggers with thresholds on the photon transverse energy $\left(E_{\mathrm{T}}^{\gamma}\right)$ ranging from $15 \mathrm{GeV}$ to $35 \mathrm{GeV}$. At the analysis level, reconstructed photons passing 
a kinematic selection are required to fulfill identification criteria and to be isolated. A data-driven double-sideband method is used to estimate contributions of background processes. The purities of the selected photon sample estimated with this method vary from $45 \%$ at $E_{\mathrm{T}}^{\gamma} \approx 20 \mathrm{GeV}$ to $99 \%$ at $E_{\mathrm{T}}^{\gamma} \approx 200 \mathrm{GeV}$.

The nuclear modification factor, denoted here as $R_{p \mathrm{~Pb}}$, is measured as a function of $E_{\mathrm{T}}^{\gamma}$, with the results presented in Figure 1. It should be noted that the definition of $R_{p} \mathrm{~Pb}$ requires a cross-section for prompt photon production in $p p$ collisions at $\sqrt{s}=8.16 \mathrm{TeV}$. However, no direct measurement at this centre-of-mass energy exists, and the corresponding cross-sections are obtained from an extrapolation of cross-sections measured in $p p$ collisions at $\sqrt{s}=8 \mathrm{TeV}$ [6]. The measurements of $R_{p \mathrm{~Pb}}$ in three centre-of-mass pseudorapidity regions are compared to predictions based on a model incorporating the energy loss of initial-state partons [7]. The model predictions shown in Figure 1 correspond to two sets of energy-loss parameters or, alternatively, no energy loss. The data are best described by the latter prediction, which indicates that initial-state partons do not lose a substantial amount of energy in soft interactions prior to the hard scattering.

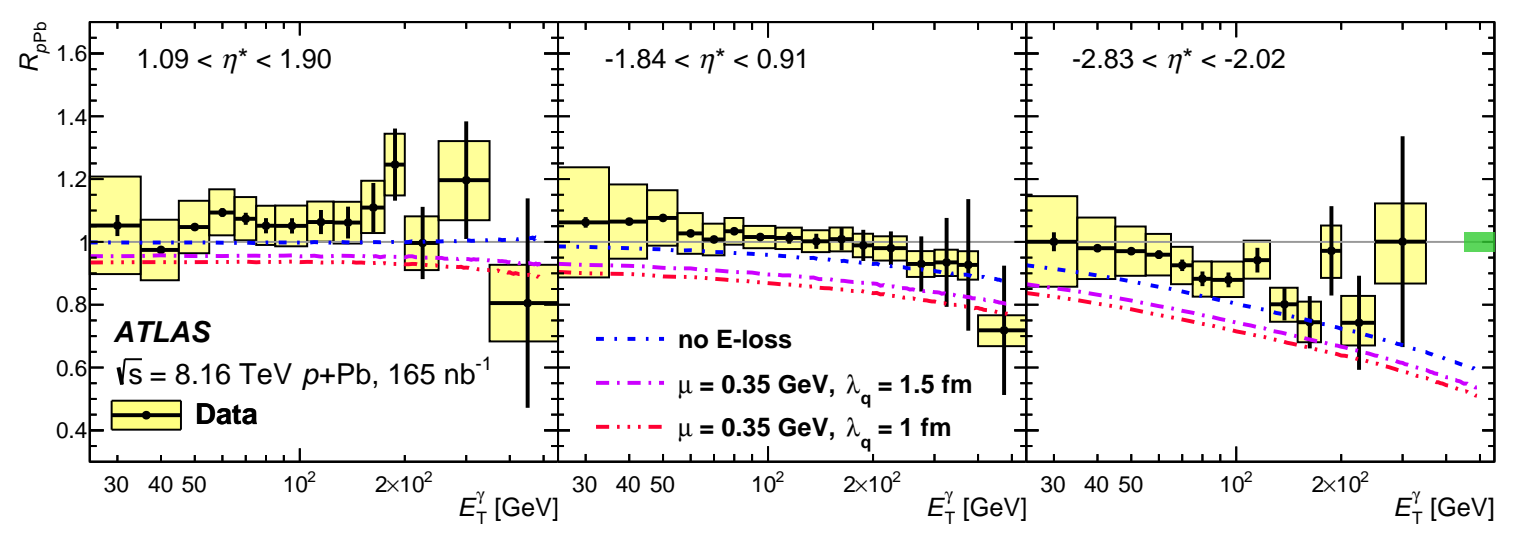

Figure 1: Nuclear modification factor $R_{p \mathrm{~Pb}}$ for isolated, prompt photons as a function of photon transverse energy $E_{\mathrm{T}}^{\gamma}$, shown for different centre-of-mass pseudorapidity, $\eta^{*}$, regions in each panel [2]. The data are compared with an initial-state energy-loss calculation. The yellow bands and vertical bars correspond to total systematic and statistical uncertainties in the data, respectively. The green box (at the far right) represents the combined $2.4 \% \mathrm{p}+\mathrm{Pb}$ and $1.9 \% \mathrm{pp}$ luminosity uncertainties.

In order to reduce the impact of systematic uncertainties in assessing the magnitude of cold nuclear matter effects, it is useful to study the ratio of $R_{p \mathrm{~Pb}}$ factors measured at forward and backward pseudorapidities. Figure 2 presents this ratio measured as a function of $E_{\mathrm{T}}^{\gamma}$. The theoretical predictions shown in comparison to data are calculated with the JETPHOX [8] code at next-to-leading order (NLO) in QCD. The PDFs used as input for the calculations come either from the free-nucleon CT14 PDF set or one of two nPDF sets: EPPS16 [10] or nCTEQ15 [11]. The free-nucleon PDF prediction is in good agreement with the data, suggesting again a lack of significant cold nuclear matter effects. However, it should be noted that the nPDF predictions are also consistent with the data within uncertainties over most of the considered $E_{\mathrm{T}}^{\gamma}$ range. 

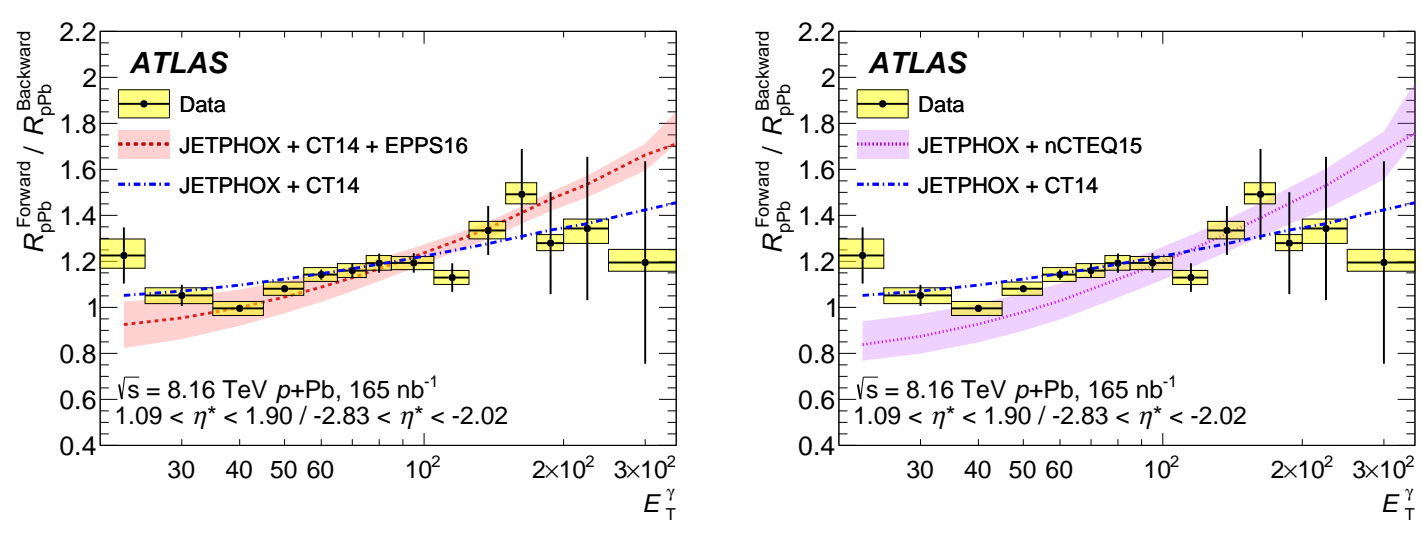

Figure 2: Ratio of the nuclear modification factor $R_{p \mathrm{~Pb}}$ between forward and backward pseudorapidity for isolated, prompt photons as a function of photon transverse energy $E_{\mathrm{T}}^{\gamma}$ [2]. The data are compared with the expectations based on JETPHOX with the EPPS16 nuclear PDF set (left) or with the nCTEQ15 nuclear PDF set (right). The yellow bands and vertical bars correspond to total systematic and statistical uncertainties in the data, respectively. The red and purple bands correspond to the systematic uncertainties in the calculations.

\section{Measurements of $W$ and $Z$ boson production in $p p$ and $\mathrm{Pb}+\mathrm{Pb}$ collisions}

The measurements of $W$ and $Z$ boson production in $p p$ and $\mathrm{Pb}+\mathrm{Pb}$ collisions use the electron and muon decay channels of the bosons $(W \rightarrow \ell v, Z \rightarrow \ell \ell$, where $\ell=e, \mu$ ). Candidate events are selected using single-lepton triggers with thresholds on the lepton transverse momentum ranging from $8 \mathrm{GeV}$ to $15 \mathrm{GeV}$. Reconstructed leptons are required to pass a kinematic selection, as well as identification and isolation requirements. Candidate events for $W$ boson production are selected by requiring exactly one good lepton and a large missing transverse energy, $E_{\mathrm{T}}^{\mathrm{miss}}>$ $25 \mathrm{GeV}$, and transverse mass, $m_{\mathrm{T}}>40 \mathrm{GeV}$. For $p p$ collisions, these quantities are calculated using the hadronic recoil reconstructed from particle flow objects, while in $\mathrm{Pb}+\mathrm{Pb}$ collisions only charged-particle tracks are used. Candidate events for $Z$ boson production are required to contain an oppositely charged lepton pair with an invariant mass in the range $66<m_{\ell \ell}<116 \mathrm{GeV}$. Background contributions from electroweak and top-quark processes are estimated using Monte Carlo simulations of these processes, while the multi-jet background contribution is evaluated with a data-driven method. In peripheral $\mathrm{Pb}+\mathrm{Pb}$ collisions, the additional contamination from electromagnetic processes, such as photonuclear interactions, is suppressed by a combination of requirements on rapidity gaps and energy deposits in Zero Degree Calorimeters.

Figure 3 presents normalised production yields for $W^{+}$and $Z$ bosons measured differentially in the charged lepton pseudorapidity and dilepton rapidity, respectively. The measured yields are compared to several theoretical predictions calculated at NLO in QCD with the MCFM code [12]. Again, the CT14 free-nucleon PDF set (including the isospin effect [13]), as well as the EPPS16 and nCTEQ15 nPDF sets, are used as input for the calculations. In all cases, the predictions are able to reproduce the shape of the data distributions reasonably well, but fail to describe their normalisation correctly. While the free-nucleon predictions underestimate the data yields only by a few percent, the nPDF predictions deviate by $10-20 \%$.

Nuclear modification factors for $W$ and $Z$ boson production are shown in Figure 4. The precise 

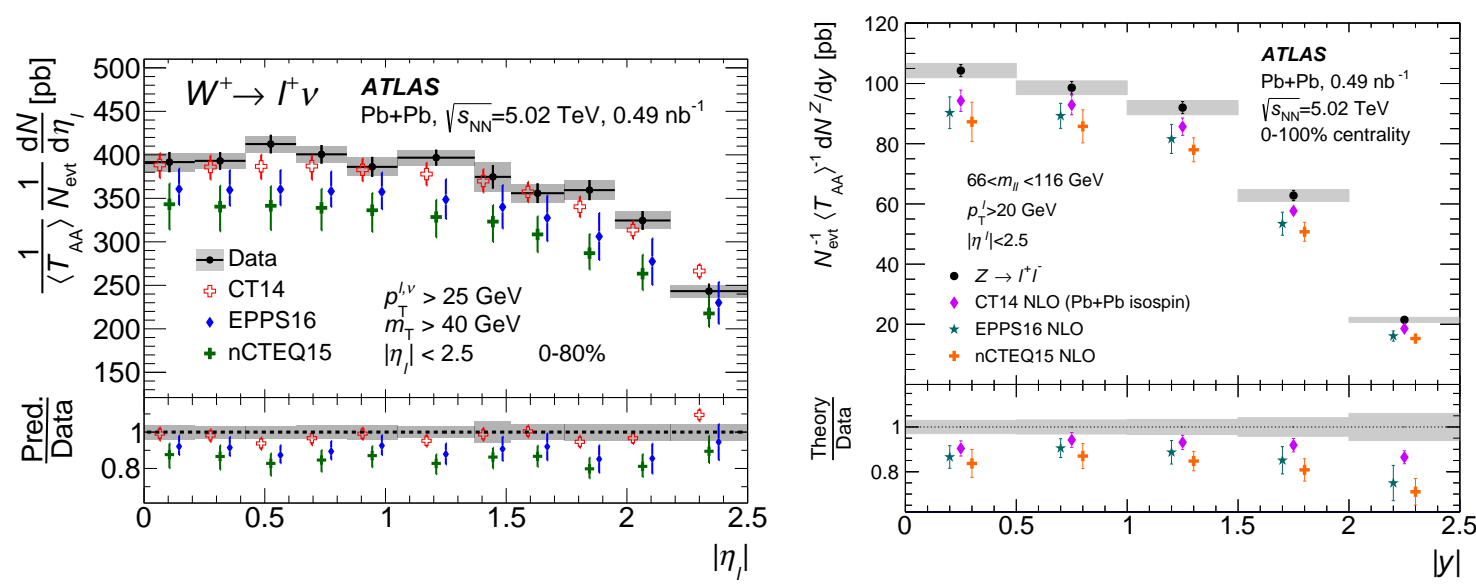

Figure 3: Nuclear modification factor $R_{\mathrm{AA}}$ obtained from the fiducial $W^{+}, W^{-}$(left) and $Z$ boson (right, bottom panel) production yields as a function of $\left\langle N_{\text {part }}\right\rangle[4,5]$. Error bars show statistical uncertainties, whereas systematic uncertainties are shown as the boxes around the data points. In the left plot, the systematic uncertainties due to $\left\langle T_{\mathrm{AA}}\right\rangle$ and luminosity of the $p p$ dataset are not included in those boxes and are shown as separate shaded boxes plotted to the right of the data points for better visibility.

cross-sections measured in $p p$ collisions [3] are used to derive the $R_{\mathrm{AA}}$ factors, which are measured as a function of the average number of nucleons participating in the collision, $\left\langle N_{\text {part }}\right\rangle$, or in centrality intervals (the two choices are equivalent). The data are compared to predictions based either on the Glauber model (constant with $\left\langle N_{\text {part }}\right\rangle$ ) [14] or on the HG-Pythia model (including a centrality bias) [15]. Since the measured $R_{\mathrm{AA}}$ factors do not depend significantly on centrality, the data are in good agreement with the Glauber model and incompatible with the HG-Pythia model.
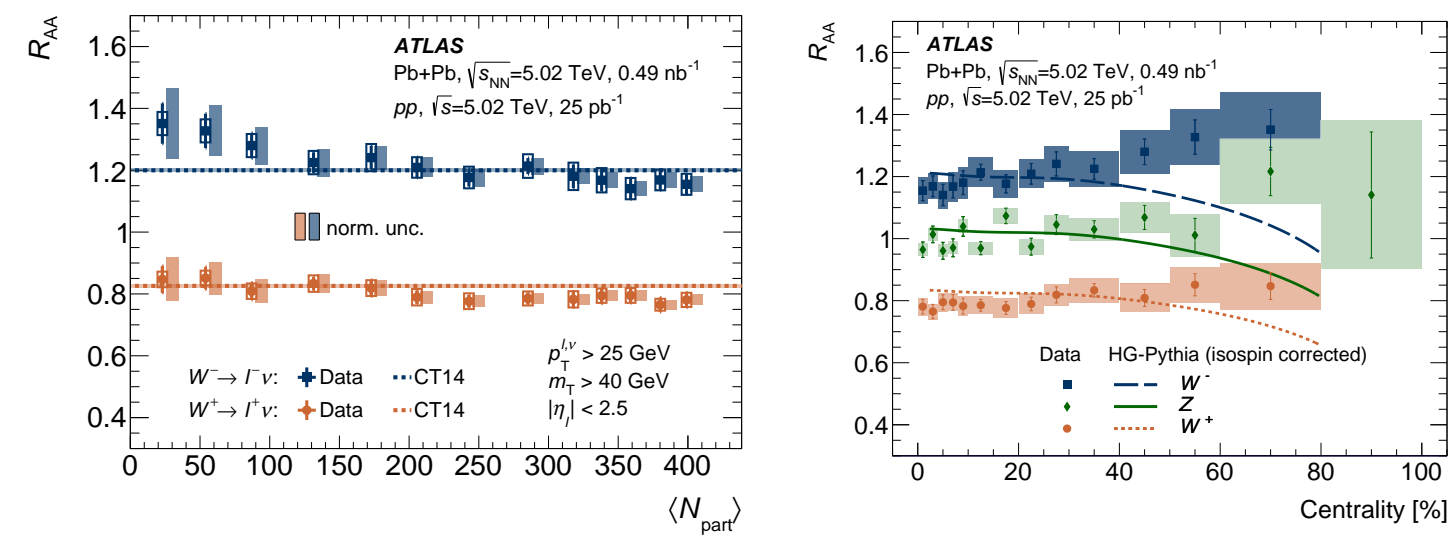

Figure 4: Nuclear modification factor $R_{\mathrm{AA}}$ obtained from the fiducial $W^{+}, W^{-}$and $Z$ boson production yields as a function of $\left\langle N_{\text {part }}\right\rangle$ (left) [4] and centrality intervals (right) [5]. Error bars show statistical uncertainties, whereas systematic uncertainties are shown as the boxes around the data points. In the left panel, the systematic uncertainties due to $\left\langle T_{\mathrm{AA}}\right\rangle$ and luminosity of the $p p$ dataset are not included in those boxes and are shown as separate shaded boxes plotted to the right of the data points for better visibility. In the right panel, the data are compared with the HG-Pythia model scaled by the isospin factors obtained from the CT14 NLO PDF calculation. 


\section{Summary}

The measurement of prompt photon production in $p+\mathrm{Pb}$ collisions at $\sqrt{s_{\mathrm{NN}}}=8.16 \mathrm{TeV}$ with the ATLAS detector at the LHC provides a study of the impact of cold nuclear matter effects on electroweak boson production. The comparison of data with predictions incorporating these effects indicates a limited impact on prompt photon production. While predictions using nPDFs are mostly consistent with the data, the best agreement is found for predictions using free-nucleon PDFs. Similarly, any significant energy loss of partons in the initial state is disfavoured by the data.

In $\mathrm{Pb}+\mathrm{Pb}$ collisions at $\sqrt{s_{\mathrm{NN}}}=5.02 \mathrm{TeV}, W$ and $Z$ boson production measurements with a precise reference from $p p$ collisions allow to study in detail models describing the collision geometry and nPDFs. The normalised $W$ and $Z$ boson production yields measured as a function of rapidity show a fairly good description of the data by a free-nucleon PDF prediction, while nPDF predictions significantly underestimate the measured yields. Nuclear modification factors for $W$ and $Z$ boson production are largely independent of the collision centrality, which is consistent with Glauber model predictions, but incompatible with predictions from the HG-Pythia model including a centrality bias.

\section{References}

[1] ATLAS Collaboration, JINST 3 (2008) S08003.

[2] ATLAS Collaboration, Phys. Lett. B 796 (2019) 230.

[3] ATLAS Collaboration, Eur. Phys. J. C 79 (2019) 128, Erratum: Eur. Phys. J. C 79 (2019) 374.

[4] ATLAS Collaboration, Eur. Phys. J. C 79 (2019) 935.

[5] ATLAS Collaboration, Phys. Lett. B 802 (2020) 135262.

[6] ATLAS Collaboration, JHEP 08 (2016) 005.

[7] Z.-B. Kang, I. Vitev and H. Xing, Phys. Rev. C 92 (2015) 054911.

[8] P. Aurenche, J. Ph. Guillet, E. Pilon, M. Werlen and M. Fontannaz, Phys. Rev. D 73 (2006) 094007.

[9] S. Dulat et al., Phys. Rev. D 93 (2016) 033006.

[10] K. J. Eskola, P. Paakkinen, H. Paukkunen and C. A. Salgado, Eur. Phys. J. C 77 (2017) 163.

[11] K. Kovařík et al., Phys. Rev. D 93 (2016) 085037.

[12] J. M. Campbell, R. K. Ellis and W. T. Giele, Eur. Phys. J. C 75 (2015) 246.

[13] P. Paakkinen, K. J. Eskola and H. Paukkunen, Phys. Lett. B 768 (2017) 7.

[14] M. L. Miller, K. Reygers, S. J. Sanders and P. Steinberg, Ann. Rev. Nucl. Part. Sci. 57 (2007) 205.

[15] C. Loizides and A. Morsch, Phys. Lett. B 773 (2017) 408. 